\title{
Development and Validation of the Psychological Well- Being Scale for Undergraduates in China
}

\author{
Changhong $\mathrm{Xia}^{1}$ Chengchieh $\mathrm{Li}^{1}{ }^{1 *}$ \\ ${ }^{1}$ School of Education, Zhaoqing University, Guangdong, China
${ }^{*}$ Corresponding author. Email: 1010765988@qq.com

\begin{abstract}
The purpose of this study was to validate the Chinese version of the psychological well-being (PWB) scale for undergraduates in China. A total of 704 participants were recruited for the current study. The results of this study showed that all items of PWB scale were medium difficulty, were suitable for undergraduates in China, and were productive to PWB. Second, all items existed measurement equivalence across sex. Third, the six-factor model of PWB was supported with adequate multiple model-fit indices. Finally, we suggested that future research could use the unidimensional model of PWB by item parceling approach in the structural equation modeling.
\end{abstract}

Keywords: psychological well-being, Rasch model, partial credit model, differential item functioning

\section{INTRODUCTION}

Based on positive psychology, well-being is regarded as a positive subjective experience in life and has become a popular and important research topic in the academy. Originally, the concept of well-being is originated form two different ancient philosophical perspective, hedonism and eudaimonism, resulting in two distinct concepts: subjective well-being and psychological wellbeing [1]. On the one hand, subjective well-being is based on hedonic perspective from Epicurus. They proposed that individuals would avoid pains and seek for happiness in life. In the eighteenth and nineteenth centuries, the thought of hedonism was connected with Locke's empiricism through individual sensory perception as the source of experience. Therefore, hedonic well-being developed a bottom-up model of the subjective wellbeing, composed of three components, including positive affect, negative affect, and life satisfaction [1].

On the other hand, psychological well-being came from eudaimonic perspective from Aristotelians. They thought that individuals would actively pursue the realization of their potential development. Afterwards, the argument of eudaimonia is consistent with Kant's rationalism to encourage individuals to strive for ideal realization or moral perfection. They advocated that human beings have an innate existence to explain life experience. Consequently, eudaimonic well-being formed a top-down model of the subjective well-being, claiming that well-being should pay more attention to personal potential development and self-realization [1]. Due to the different philosophical perspectives, the concepts, characteristics and measurement indicators of both subjective and psychological well-being are derived. Subjective well-being includes more affections and overall evaluation for quality of life while psychological well-being examines individual's feelings about current life challenges, such as meaningful goal pursuit, personal growth and development, and building social relationships with others [2].

In practice, Diener first proposed subjective wellbeing as the academic term, and developed a five-item satisfaction with life scale with his colleagues [3]. However, some studies criticized that the definition of Diener's subjective well-being is not rigorous enough, and there is no theoretical basis for the connotation of subjective well-being [4]. Therefore, Ryff paid more emphasis on eudaimonic perspective into subjective well-being and proposed psychological well-being as the academic term [2].

Ryff integrated Erikson's personal development, Buhler's basic life orientation, Frankl's pursuit of meaning, Jahoda's mental health, Jung's individualization, Maslow's self-realization, Neugarten's personality styles, Rogers' fully functional people, and Allport's maturity into psychological well-being [4]. She developed a multiple dimensional psychological wellbeing model, including self-acceptance, positive 
relations with others, personal growth, purpose in life, environmental mastery, and autonomy.

In the beginning, Ryff developed a self-report 120item psychological well-being scale. Six dimensions of positive human health were assessed and each dimension was assessed by a set of 20 items [4]. Later, Ryff revised the longer version to form the 84-item version [5], but the shorter one has been criticized because the subscales aren't clearly divided into six factors by exploratory factor analysis. Moreover, participants may even feel burdened when filling the questionnaire. Finally, she decreased the items into an 18 -item version [6]. Some studies found the internal consistency reliability of the parsimonious version was not adequate that though the model-fit indices of the six-factor model and the hierarchical model were acceptable [7]. Therefore, there were a various of versions developed in many countries based on Ryff's psychological well-being scale to retest its validation. For example, Li retranslated the 84-item version, and used confirmatory factor analysis to select items, and then form an 18-item Chinese version [8]. Later, $\mathrm{Li}$ and his advisor ensured that this scale had good reliability, validity and measurement invariance for college students in Taiwan [9].

Thus, the purpose of this study was to investigate the validity of the psychological well-being scale $\mathrm{Li}$ and $\mathrm{Yu}$ developed with a sample of undergraduates in China. In this study, we conducted Rasch analysis to estimate the difficulties and model-data fits of items and to test the measurement invariance across sex for each item by differential item functioning. Second, we employed confirmatory factor analysis to construct the validation and competition model of psychological well-being.

\section{METHOD}

\subsection{Participants}

There were 704 undergraduates from a public university in China who took the course of psychological development and health. There were 574 female and 130 male students. Because the participants were normal students from a various of departments, the female students were larger then male.

\subsection{Measures}

We used four kinds of scales, including psychological well-being scale, subjective well-being scale, psychological capital scale, and mental health lifestyle scale. First, the subjective well-being scale was employed to measure the participants' subjective wellbeing in this study [10], including psychological, social, and affective well-beings. The scale consists of fifteen items, its internal consistency was 0.762 , and model it indices were adequate $\left(\chi^{2}(87)=808.791, p<0.05\right.$; CFI $=$ 0.762 ; TLI $=0.713 ;$ RMEA $=0.109 ;$ SRMR $=0.093)$.
Participates rated items on a 5-point Likert-type scale ranging from "strongly disagree" to "strongly agree".

Second, the psychological capital scale was employed to measure the participants' psychological capital in this study [11], including self-efficacy, hope, resilience, and optimism. The scale consists of 24 items, its internal consistency was 0.938 , and model it indices were adequate $\left(\chi^{2}(246)=1413.709, p<0.05 ; \mathrm{CFI}=0.864\right.$; $\mathrm{TLI}=0.847 ;$ RMEA $=0.082 ;$ SRMR $=0.055$ ). Participates rated items on a 5-point Likert-type scale ranging from "strongly disagree" to "strongly agree".

Finally, the mental health lifestyle scale was employed to measure the participants' mental health lifestyle in this study [12], including activities, life satisfaction, and depression. First, the subscale for activities consists of four items, its internal consistency was 0.724. Participates made their judgements of the experiences during the past month on a 5-point Likerttype scale ranging from "completely no" to "almost every day". Second, the subscale for life satisfaction consists of six items, its internal consistency was 0.776 . Participates made their judgements of the feelings during the past month on a 5-point Likert-type scale ranging from "strongly dissatisfactory" to "strongly satisfactory". Third, the subscale for depression consists of five items, its internal consistency was 0.879 . Participates made their judgements of the feelings during the past week on a 5-point Likert-type scale ranging from "completely no" to "strongly serious".

\subsection{Analysis}

We conducted Rasch analysis and confirmatory factor analysis (CFA) to construct the validation of psychological well-being (PWB) scale. On the one hand, we employed the partial credit model in the Rasch family to estimate the difficulties and model-data fits of items, and used the differential item functioning (DIF) to test the measurement invariance across sex for each item by ConQuest software with the marginal maximum likelihood method. On the other hand, we employed CFA to examine the psychometric validity and to see which model would yield the best model fit by Mplus software with the maximum likelihood method. The first model is a six-factor construct of PWB scale underpinned by positive relations with others, autonomy, environmental mastery, personal growth, purpose in life, and selfacceptance. Model 2 proposes the hierarchical model of PWB scale. Model 3 is a unidimensional model of PWB scale wherein all constructs loaded onto a single manifest item by item parceling approach. Finally, we investigated how psychological well-being was associated with subjective well-being, psychological capital, mental health lifestyle, life satisfaction, and depression. 


\section{RESULT}

Prior to Rasch analysis and CFA, the descriptive statistics of each item for PWB scale, including the mean, standard deviation, skewness, and kurtosis, were suitable for normal distribution.

\subsection{Rasch analysis}

Table 1 presented the item difficulty estimates, model-data fit test, and estimates of DIF across sex of PWB scale. With regard to item difficulty, the estimates for PWB were adequate ranging from -0.901 to 0.757 logits, and half of them were negative numbers. It indicated that all items of PWB scale were medium difficulty and were suitable for undergraduates in China. In terms of model-data fit, both of the weighted and unweighted mean squares (MNSQ) were acceptable ranging from 0.54 to 1.53 . It demonstrated that all items were productive to PWB scale. Regarding DIF, the differences of all item difficulty across sex were less than 0.426 logits [13]. It showed that all items didn't have a bias against sex while male and female participants fill this scale.

Table 1. Results of PCM of Rasch analysis and DIF for psychological well-being scale

\begin{tabular}{|c|c|c|c|c|c|c|c|}
\hline \multirow[b]{2}{*}{ Item } & \multirow[b]{2}{*}{ Difficulty } & \multirow[b]{2}{*}{ SE } & \multicolumn{2}{|c|}{ Unweighted } & \multicolumn{2}{|c|}{ Weighted } & \multirow[b]{2}{*}{ DIF } \\
\hline & & & MNSQ & $t$ value & $\overline{M N S Q}$ & $t$ value & \\
\hline 1 & 0.081 & 0.028 & 1.53 & 8.6 & 1.46 & 7.8 & 0.096 \\
\hline 2 & 0.462 & 0.030 & 1.09 & 1.7 & 1.08 & 1.5 & 0.028 \\
\hline 3 & -0.298 & 0.031 & 1.07 & 1.3 & 1.05 & 1.9 & 0.118 \\
\hline 4 & 0.415 & 0.030 & 1.03 & 0.5 & 1.03 & 0.5 & 0.284 \\
\hline 5 & 0.585 & 0.030 & 1.07 & 1.3 & 1.08 & 1.5 & 0.252 \\
\hline 6 & -0.113 & 0.030 & 1.03 & 0.5 & 1.03 & 0.6 & 0.124 \\
\hline 7 & -0.160 & 0.031 & 0.83 & -3.4 & 0.54 & -3.3 & 0.016 \\
\hline 8 & -0.594 & 0.032 & 0.86 & -2.7 & 0.88 & -2.5 & 0.130 \\
\hline 9 & -0.229 & 0.030 & 1.13 & 2.4 & 1.12 & 2.2 & 0.004 \\
\hline 10 & -0.577 & 0.031 & 1.06 & 1.2 & 1.04 & 0.8 & 0.114 \\
\hline 11 & -0.901 & 0.033 & 0.90 & -1.8 & 1.00 & -0.0 & 0.234 \\
\hline 12 & -0.782 & 0.033 & 0.96 & -0.8 & 0.99 & -0.1 & 0.278 \\
\hline 13 & 0.227 & 0.030 & 0.97 & -0.6 & 0.98 & -0.4 & 0.012 \\
\hline 14 & 0.224 & 0.030 & 0.90 & -1.9 & 0.91 & -1.8 & 0.120 \\
\hline 15 & -0.142 & 0.030 & 1.17 & 3.1 & 1.14 & 2.3 & 0.180 \\
\hline 16 & 0.757 & 0.030 & 0.98 & -0.4 & 0.98 & -0.3 & 0.008 \\
\hline 17 & 0.501 & 0.030 & 0.84 & -3.2 & 0.84 & -3.2 & 0.164 \\
\hline 18 & 0.543 & 0.123 & 0.89 & -2.1 & 0.89 & -2.1 & 0.156 \\
\hline
\end{tabular}

\subsection{Confirmatory factor analysis}

Table 2 showed the standardized estimates, squared multiple correlation (SMC) and convergent validity of the six-factor model for PWB scale. The parameters in the model were significant. Although the chi square test rejected the model $\left(\chi^{2}(120)=405.776, p<.05\right)$, multiple indices to evaluate the goodness of model fit were adequate $(\mathrm{CFI}=0.949>0.90, \mathrm{TLI}=0.936>0.90$, $\mathrm{RMSEA}=0.058<0.08$, SRMR $=0.038<0.05)$, suggesting that this model was acceptable. In terms of convergent validity, the component reliabilities (CR) were perfect ranging from 0.713 to 0.841 higher than 0.60 , and the average variance extracted (AVE) were adequate ranging from 0.455 to 0.640 . Regarding the discriminant validity, the square roots of AVE for all latent constructs were higher than the correlation coefficients among all latent constructs. It meant that all latent constructs of PWB scale were discriminant from each other. With regard to criterion-related validity, psychological well-being was correlated with subjective well-being, psychological capital, mental health lifestyle, life satisfaction, and depression. Psychological wellbeing was significantly positively related to subjective well-being $(r=0.577)$, psychological capital $(r=0.767)$, mental health lifestyle $(r=0.241)$, and life satisfaction $(r$ $=0.564)$. On the contrary, it was significantly negatively associated with depression $(r=-0.204)$.

Table 2. Results of confirmatory factor analysis for psychological well-being scale

\begin{tabular}{|c|c|c|c|c|}
\hline Item & Factor loading & SMC & CR & AVE \\
\hline $\mathbf{1}$ & 0.584 & 0.341 & 0.713 & 0.455 \\
\hline $\mathbf{2}$ & 0.734 & 0.538 & & \\
\hline
\end{tabular}




\begin{tabular}{|c|c|c|c|c|}
\hline $\mathbf{3}$ & 0.696 & 0.485 & & \\
\hline $\mathbf{4}$ & 0.728 & 0.530 & 0.752 & 0.503 \\
\hline $\mathbf{5}$ & 0.692 & 0.479 & & \\
\hline $\mathbf{6}$ & 0.708 & 0.501 & & 0.599 \\
\hline $\mathbf{7}$ & 0.782 & 0.612 & 0.816 & \\
\hline $\mathbf{8}$ & 0.852 & 0.726 & & 0.539 \\
\hline $\mathbf{1 0}$ & 0.677 & 0.458 & & 0.778 \\
\hline $\mathbf{1 1}$ & 0.699 & 0.488 & & 0.632 \\
\hline $\mathbf{1 2}$ & 0.760 & 0.577 & & 0.835 \\
\hline $\mathbf{1 3}$ & 0.743 & 0.552 & & \\
\hline $\mathbf{1 5}$ & 0.838 & 0.702 & & \\
\hline $\mathbf{1 6}$ & 0.866 & 0.751 & & \\
\hline $\mathbf{1 7}$ & 0.665 & 0.442 & & \\
\hline $\mathbf{1 8}$ & 0.728 & 0.529 & & \\
\hline
\end{tabular}

Finally, we conducted a series of CFA models to examine which one would yield the best fit by model competition. Model 1 was a six-factor construct of PWB scale, Model 2 was the hierarchical model of PWB scale, and Model 3 was a unidimensional model of PWB scale by item parceling approach. Multiple model-fit indices of Model 1 were better than Model $2\left(\chi^{2}(129)=439.758, p\right.$ $<.05, \mathrm{CFI}=0.945, \mathrm{TLI}=0.935, \mathrm{RMSEA}=0.058, \mathrm{SRMR}$ $=0.042, \mathrm{AIC}=32747.845, \mathrm{BIC}=33021.251)$. Moreover, Multiple model-fit indices of Model $3\left(\chi^{2}(9)=33.921, p\right.$ $<.05, \mathrm{CFI}=0.983$, TLI $=0.971$, RMSEA $=0.063$, SRMR $=0.022, \mathrm{AIC}=9805.909, \mathrm{BIC}=9887.931)$ were better than Model 1 and Model 2. It indicated that Model 3 was the most parsimonious model.

\section{CONCLUSION}

Overall, results of this study indicated that the PWB scale was an acceptable psychological well-being measure. These findings supported the factorial and construct validity as well as reliability of the PWB scale, which may be a proactive approach to understand individual comprehensive psychological well-being for undergraduates in China. The items of the PWB scale were medium difficulty and existed measurement equivalence across sex. Moreover, the individual item score of the PWB scale could be calculated into a total score based on item response theory. Therefore, we suggested that future research could use six-factor model or the parsimonious model of PWB scale by item parceling approach in the structural equation modeling.

\section{REFERENCES}

[1] R.M. Ryan, E.L. Deci, On happiness and human potentials: A review of research on hedonic and eudaimonic well-being, Annual Review of Psychology 52(1), 2001, pp. 141-166.

[2] C.L.M. Keyes, D. Shmotkin, C.D. Ryff, Optimizing well-being: The empirical encounter of two traditions, Journal of Personality and Social Psychology, 82(6), 2002, pp. 1007-1022.

[3] E. Diener, R.A. Emmons, R.J. Larsen, S. Griffin, The satisfaction with life scale, Journal of Personality Assessment, 49(1), 1985, pp. 71-75.
[4] C.D. Ryff, Happiness is everything, or is it? Explorations on the meaning of psychological wellbeing, Journal of Personality and Social Psychology, 57(6), 1989, pp. 1069-1081.

[5] C.D. Ryff, Y.H. Lee, M.J. Essex, P.S. Schmutte, My children and me: Mid-life evaluations of grown children and of self, Psychology and Aging, 9(2), 1994, pp. 195-205.

[6] C.D. Ryff, C.L.M. Keyes, The structure of psychological well-being revisited, Journal of Personality and Social Psychology, 69(4), 1995, pp. 719-727.

[7] S.T. Cheng, A.C.M. Chan, Measuring psychological well-being in the Chinese, Personality and Individual Differences, 38(6), 2005, pp. 1307-1316.

[8] R.H. Li, Reliability and validity of a shorter Chinese version for Ryff's psychological well-being scale. Health Education Journal, 73(4), 2014, pp. 446-452.

[9] R.H. Li, M.N. Yu, Reliability, validity, and measurement invariance of the brief Chinese version of psychological well-being scale among college students, Chinese Journal of Guidance and Counseling, 46, 2016, pp.127-154.

[10] M.N. Yu, P.L. Chen, Y.H. Chen, Study of scale-items reduction: The reconstruction of subjective wellbeing scale, Journal of Educational Research and Development, 13(4), 2017, pp.27-56.

[11] M.N. Yu, P.L. Chen, Y.F. Tang, The construction and application of psychological capital scale for college students, Journal of Educational Research and Development, 8(4), 2012, pp.19-52.

[12] C.C. Li, Y. Tang, S.M. Li, T.Y. Tsai, Type of mental health lifestyle required in Taiwan, Taiwan J Psychiatry 34(3), 2020, pp. 134-137.

[13] M. Wilson. Constructing measures: An item response modeling approach. New Jersey: Lawrence Erlbaum associates, 2005. 